\title{
Myocardial Delineation via Registration in a Polar Coordinate System
}

\author{
Nicholas M.I. Noble ${ }^{1}$, Derek L.G. Hill ${ }^{1}$, Marcel Breeuwer ${ }^{2}$, Julia A. Schnabel ${ }^{1}$, \\ David J. Hawkes ${ }^{1}$, Frans A. Gerritsen ${ }^{2}$, and Reza Razavi ${ }^{1}$ \\ ${ }^{1}$ Computer Imaging Science Group, Guy's Hospital, Kings College London, UK \\ 2 Philips Medical Systems, Medical Imaging Information Technology - Advanced Development
}

\begin{abstract}
This paper describes a technique for automatic analysis of dynamic magnetic resonance images of the left ventricle using a non-rigid registration algorithm. Short axis cine images were re-sampled into polar coordinates before all the time frames were aligned using a non-rigid registration algorithm. An manually delineated contour of a single phase was propagated through the dynamic sequence. Two variants of this approach were investigated and compared with manual delineation of all phases, and with the commercially available automatic MASS package. The results showed good correlation with manual delineation and produced fewer erroneous results than the commercial package.
\end{abstract}

\section{Introduction}

Cardiac magnetic resonance imaging (MRI) can now provide high quality images of the heart, including myocardial contraction, perfusion and with late enhancement, information on myocardial viability. A major obstacle to the widespread clinical use of cardiac MRI, however, is the difficulty in analysing the images produced [1]. To combine all of this information into a "one-stop-shop" assessment of patients with ischeamic heart disease requires a great deal of analysis, which when performed manually, is very time consuming. A rapid automated method of deriving information from these images would greatly facilitate their use. This paper addresses the problem of automatic analysis of short axis cine MR images in order to assess left ventricular function. The technique is potentially extendible to the analysis of data from an entire cardiac MR examination.

Quantitative measures of global left ventricular function (eg: ejection fraction and ventricular mass) and regional left ventricular function (eg: wall motion, wall thickness and wall thickening) can be calculated from segmented surfaces of the epicardium and endocardium. In our experience, current techniques for automatically delineating these surfaces from MR images are insufficiently robust for routine clinical use. Automatic techniques tend to fail around the papillary muscle or where pathology is present. As a consequence, the analysis of ventricular function currently requires substantial input from a skilled operator to perform manual delineation or manual correction of contours.

Segmentation techniques that are solely image driven, such as those based on image gradients alone, are unable to extract reliable myocardial surfaces [2], especially: in the presence of the papillary muscles and near the apex. Model based techniques that incorporate a priori knowledge into the segmentation process [3] [4] show good 
promise, however the substantial variation in size and shape of pathological myocardium makes a comprehensive model hard to construct.

We, therefore, propose an alternative approach based on non-rigid image registration. In our current implementation, we assume that the endocardial and epicardial surfaces have been manually segmented at a single phase; end-diastole.

The intrinsic radial motion of the myocardium, suggests that registration algorithms optimised in Cartesian coordinate systems, may not be the optimal way in which to perform myocardial registration. Conventional short axis images were hence re-sampled into a polar coordinate system, and registered in that coordinate system before contour propagation was performed. The technique is compared with manual delineation and a commercial software package on a group of 10 patients.

\section{Data}

Short axis ECG triggered steady state free precession images with SENSE factor 2 (Figure 1, top row) were obtained in 10 patients undergoing cardiac MRI for the investigation of coronary artery disease. Three slices (corresponding to approximately basal, mid and apical positions) were selected from 8 - 9 contiguous slices, imaged with: slice thickness $8-10 \mathrm{~mm}$, field of view $350 \times 344-390 \times 390 \mathrm{~mm}$, image size $256 \times 256,20-25$ phases

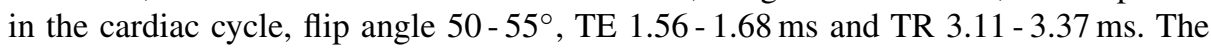
images were acquired on a Philips Gyroscan Intera $1.5 \mathrm{~T}$ with master gradients, using a 5 element cardiac synergy coil and vector ECG.

\section{Methods}

\subsection{Technique 1: Manual Delineation}

Clinical opinion is divided regarding the correct manual delineation of endocardial boundaries. In particular, different centres use different criteria for the inclusion of papiliary muscles. The manual delineation in our centre excluded papiliary muscles unless they were completely incorporated into the myocardial wall.

For every phase of each selected slice of each image set, both the epi- and endocardial contours were manually delineated in the original coordinate system. The volumes contained by the epi- and endocardial delineations were then calculated (area contained by contour $\times$ slice thickness) at each phase and for each selected slice of each image set.

\subsection{Technique 2: All-to-One Registration}

A centre of area (COA) of the left ventricular blood pool was calculated from the voxels enclosed by the manual endocardial delineation at end diastole (from technique 1) as per equation 1, where $\left(x_{i}, y_{i}\right)$ are the coordinates of the $i^{\text {th }}$ voxel enclosed by the delineation and $n$ is the total number of whole voxels contained within the delineation.

$$
\mathrm{COA}=\left(\frac{\sum_{i=1}^{n} x_{i}}{n}, \frac{\sum_{i=1}^{n} y_{i}}{n}\right) .
$$



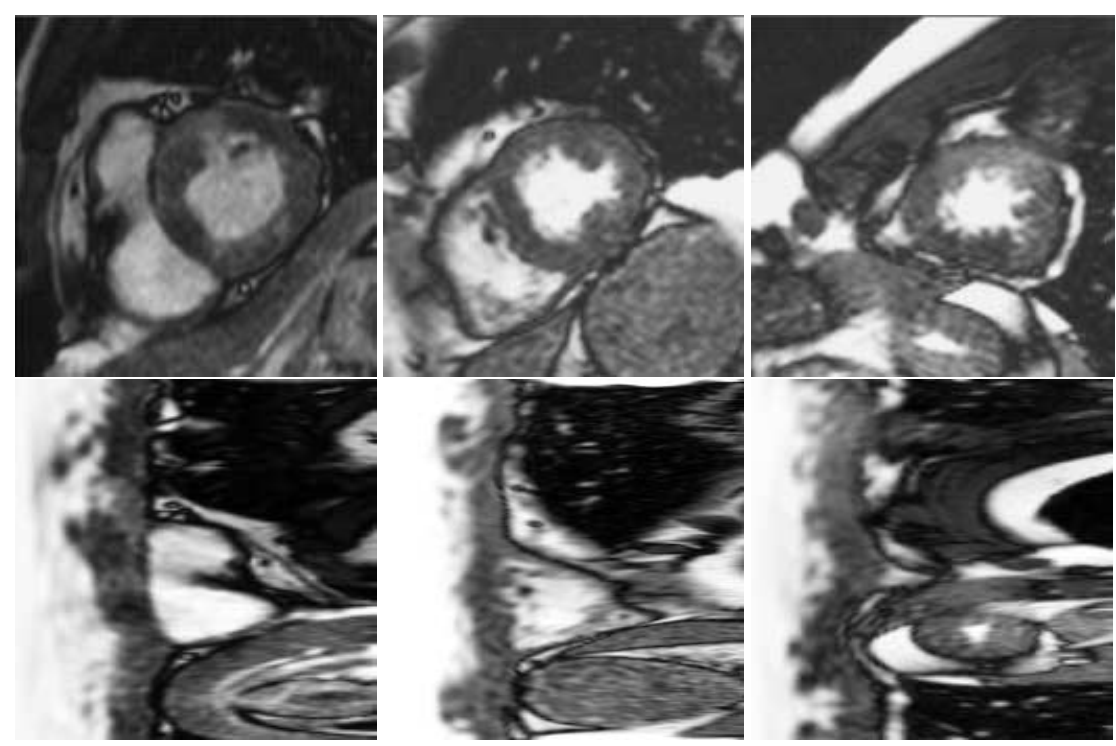

Fig. 1. Original (top row) and re-sampled images (bottom row) with intensity values for theta on the $\mathrm{y}$-axis plotted versus radial distance from the origin on the $\mathrm{x}$-axis, for basal, (left) mid (centre) and apical (right) slices. The bottom row images show from their left side; firstly the left ventricular blood pool, followed by the left ventricular myocardium

For each slice, every phase was then re-sampled in a polar fashion about the calculated COA for that slice, using bilinear interpolation with 256 eqi-spaced radial spokes, and 165 radial steps with a radial step size of 0.4 voxels.

The re-sampled images (Figure 1, second row) were then registered together in an all-to-one manner (phase 1 - phase 2, phase 1 - phase 3 , phase 1 - phase 4 ...), using two dimensional non-rigid registration (based on [5]) with normalised mutual information [6] employed as the similarity measure. The epi- and endocardial manual delineations at phase 1 were then re-sampled into the polar coordinate system, and warped according to the results of the registrations, in an all-to-one fashion, the resultant warped contours were then re-sampled into the original Cartesian coordinate system (Figure 2] top row), and the epi- and endocardial volumes were calculated at each phase and for each selected slice of each image set as per section 3.1

\subsection{Technique 3: Piecewise Registration}

Radial re-sampling was performed as per section 3.2 The images were then registered in a piecewise manner (phase 1 - phase 2 , phase 2 - phase 3 , phase 3 - phase $4 \ldots$ ), using the same non-rigid registration algorithm employed in section 3.2. The epi- and endocardial manual delineations at end-diastole were then propagated and processed as per section 3.2, except that this time the delineations were warped in a piecewise manner (Figure 2 , middle row). 


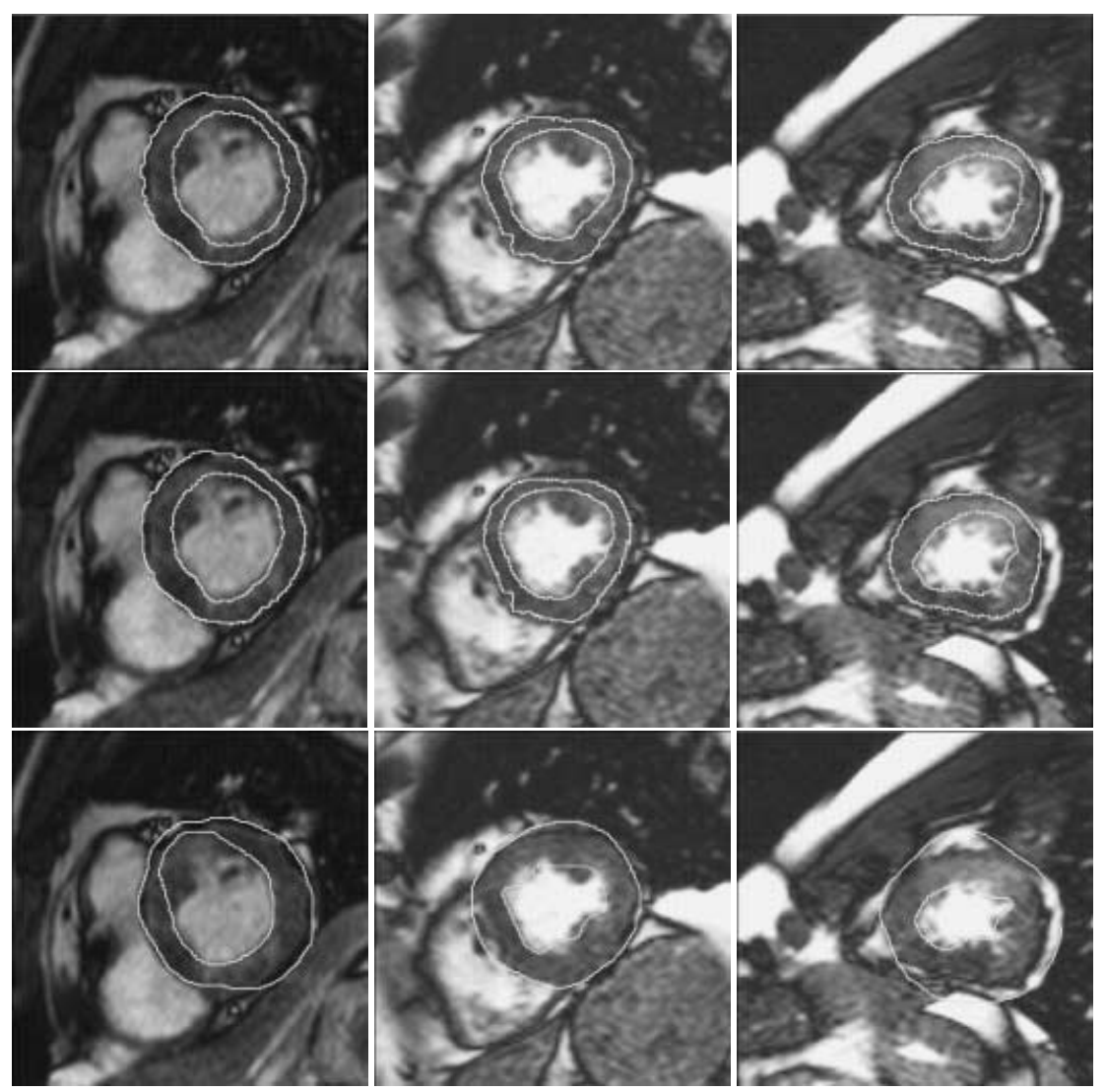

Fig. 2. Original images with overlaid delineations for techniques: 2 (top row), 3 (middle row) and 4 (bottom row), for basal (left), mid (centre) and apical (right) slices

\subsection{Technique 4: Segmentation Using Commercial Software}

Contours were automatically detected in the original coordinate system using the commercially available MASS package (Version 4.2, Medis, Leiden, the Netherlands),as described by Van der Geest et al. [7] (Figure 2] bottom row). The epi- and endocardial volumes were calculated at each phase and for each selected slice of each image set, as per section 3.1 .

\section{Results}

The correlation coefficients between epi- and endocardial volumes (Figure 3) produced from manual delineations and techniques 2, 3 and 4 were calculated. These values are shown for all ten patients in figure 4

For all three slices, the mean value of the correlation coefficient for the endocardial volume is higher for techniques 2 and 3 than for technique 4. For the apical and basal 
slices, this difference is significant at the 5\% level (paired t-test). There is however no significant difference between technique 4 and either of techniques 2 and 3 for the epicardial boundary.

It is also interesting to assess which variant of the novel technique performs best. We found no significant difference for the endocardial boundaries. However, for the epicardial boundaries, technique 3 (piecewise) had a higher correlation coefficient than technique 2 (significant at the $5 \%$ level).
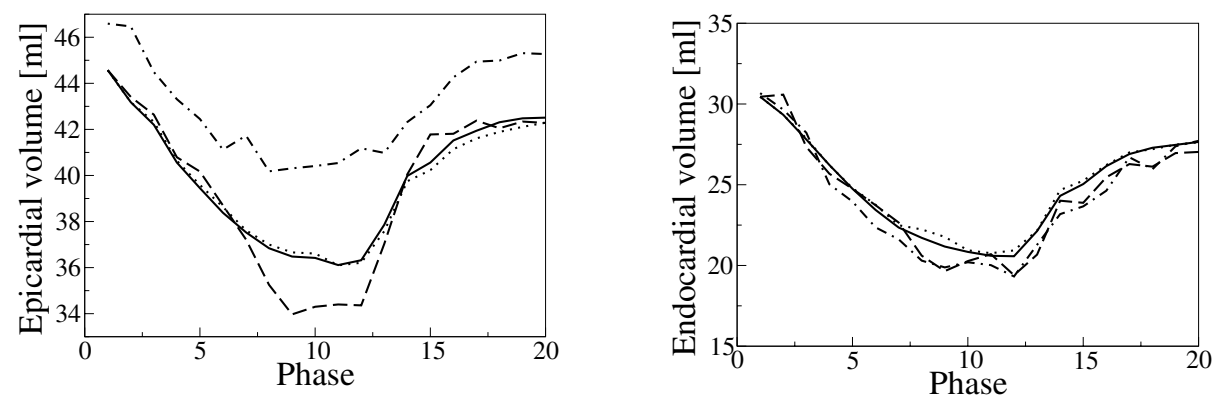

Fig. 3. Example of the epicardial (left) and endocardial (right) volumes plotted against phase in the cardiac cycle for techniques: 1 (dashed line), 2 (dotted line), 3 (solid line) and 4 (dot-dashed line) for the basal slice of a single patient

The quality of the delineations produced by the three methods was also assessed qualitatively. Each delineation was manually classified as either requiring a correction (a failure) or being plausible. The mean percentage of images per selected slice per patient (to normalise for non-uniform numbers of images per scan), that failed was calculated for techniques: 2, 3 and 4 for both epi- and endocardial contours at each selected slice (Figure 5). It can be seen from figure 5 that techniques 2 and 3 required significantly fewer manual corrections than technique 4 when detecting the endocardial contour for all slices. Figure 5 also shows that whilst techniques 2 and 3 required very little manual correction when detecting the epicardial contour, technique 4 required manual correction in $90-95 \%$ of contours detected.

\section{Discussion}

We have described a new technique for analysis of left ventricular function from short axis cine cardiac MR images. The technique delineates the epicardial and endocardial boundaries by non-rigid registration of the images across the phases of the cardiac cycle, and propagation of manually delineated boundaries at one phase using the calculated deformation field.

Manual problems. Although the results in this paper have been compared to manual delineation, this is by no means a 'gold standard' given the inherent human error in such delineations and the different techniques used especially in inclusion or exclusion 

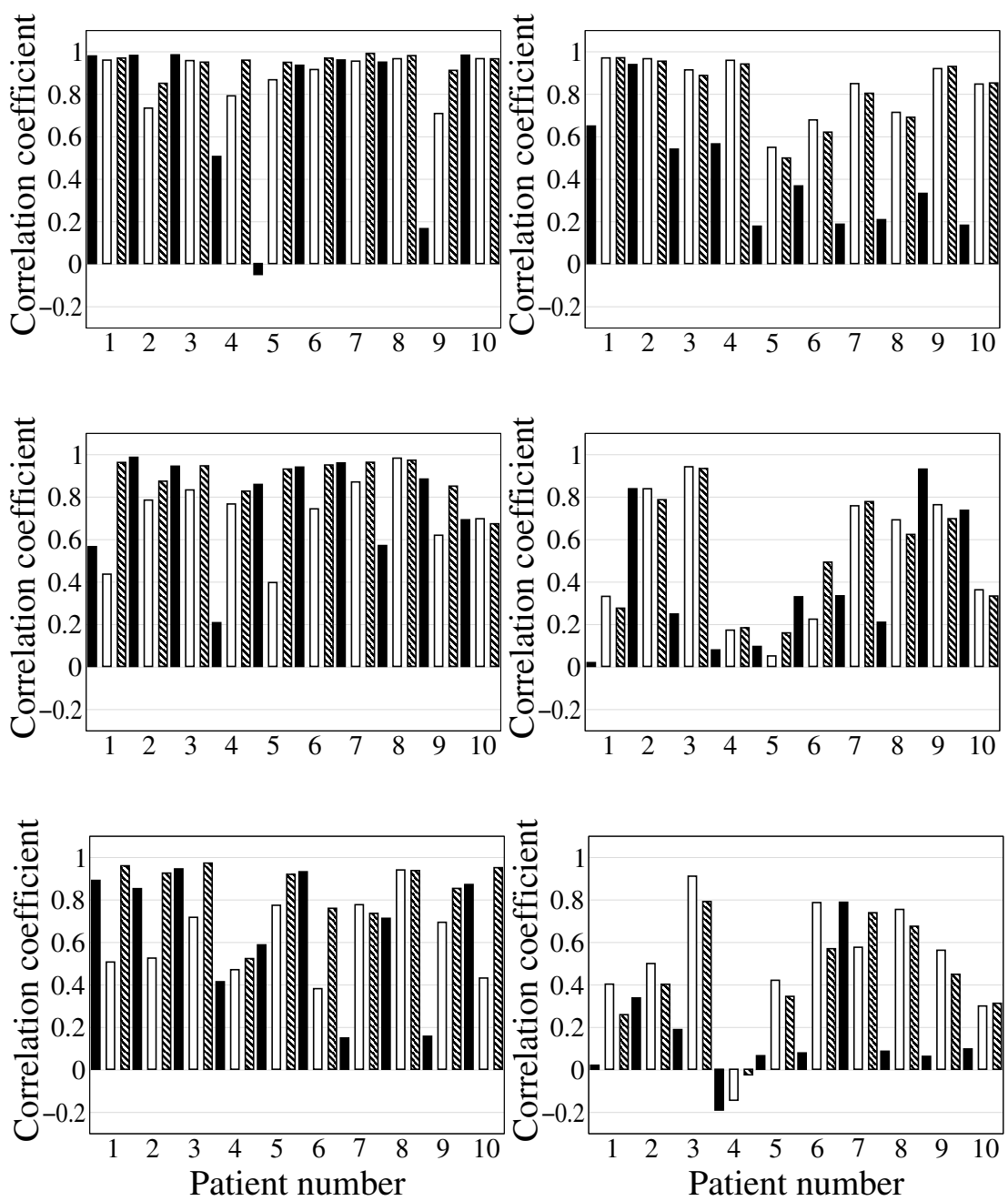

Fig. 4. Charts of the correlation of: epicardial volume (left column) and endocardial volume (right column) between manual delineation and techniques 2 (light bar), 3 (striped bar) and 4 (dark bar), for basal (top row), mid (centre row) and apical (bottom row) slices for each patient. $N B$ techniques 2, 3 and 4 failed in all phases to correctly detect the endocardial contour of the apical slice of patient 4

of papiliary muscles. Also, the sensitivity of techniques 2 and 3 to the initial contour mean that the end result is only as good as the original delineation with which they are supplied.

Endocardial contour. Propagation of the end-diastole contour used in these techniques does not take into account the papiliary muscles which can form part of the myocardial mass especially in mid slices of the ventricle at end-systole. There is therefore discrep- 

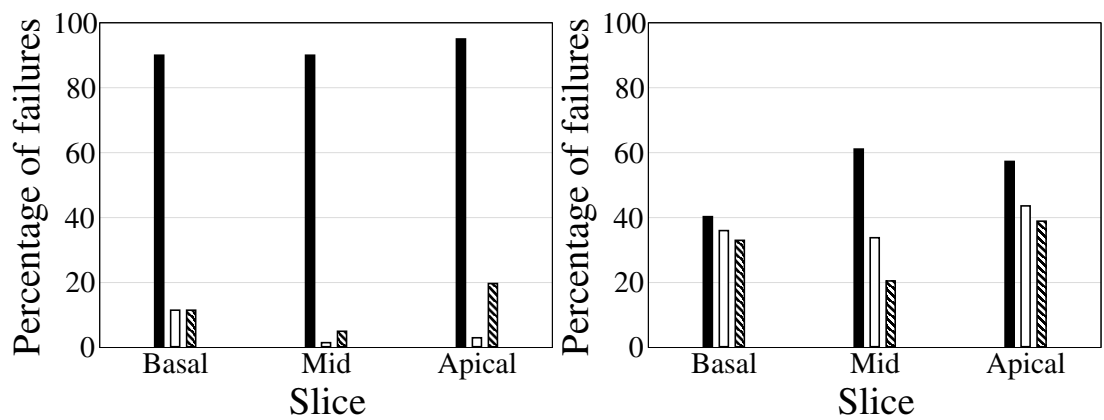

Fig. 5. Charts showing the mean percentage of images per selected slice per patient that required manual correction of the contour, for techniques: 2 (light bar), 3 (striped bar) and 4 (dark bar), for: epicardial (left) and endocardial (right) contours

ancy between techniques 2, 3 and technique 1, with techniques 2 and 3 overestimating the endocardial volume.

Through-slice motion. Throughout the cardiac cycle, papiliary muscles are constantly moving into and out-of the imaging plane. This complicates the alignment for techniques 2 and 3 as the registration algorithm attempts to align objects that are not present in both the source and target images. Manual observation of technique 3 shows that it manages to cope with such motion better than technique 2, the gradual warping of the contour making it easier to follow the endocardial contour. This problem could be solved by extending this two dimensional technique to a full three dimensional method.

Radial continuity. A lack of continuity at the edge of the re-sampled images means that two contour points which are adjacent in the original coordinate system could be warped to quite different positions after registration. Potential solutions to this could be: to wrap the registration b-splines around at the edge of the image, or to avoid the use of re-sampled images and the subsequent interpolation errors and instead implement a polar or radial control point spacing within the registration algorithm.

Quantitative comparison techniques. The validation experiments that we have performed could be complemented by a measure that incorporates the local distance between compared contours. Future work will incorporate such a technique.

\section{Conclusion}

A novel approach to the registration of cardiac magnetic resonance images has been introduced. Ten sets of patient images were re-sampled into a polar coordinate system and two registration strategies were investigated (all-to-one and piecewise). The resulting propagated contours were then compared with contours automatically created by MASS and with manual delineations. The piecewise technique correlated with manual delineation better than the all-to-one and MASS detected techniques, and also required fewer manual interactions to correct erroneously detected contours than MASS. 


\section{Acknowledgements}

We are grateful to Philips Medical Systems Nederland B.V. Medical Imaging Information Technology - Advanced Development for funding this work, and to those at the Computer Imaging Sciences Group for their assistance.

\section{References}

1. A.F. Frangi, W.J. Niessen, and M.A. Viergever. Three-Dimensional Modeling for Functional Analysis of Cardiac Images: A Review. IEEE Trans. Med. Imaging, 20(1):2-25, 2001.

2. W.J. Niessen, B.M. ter Haar Romeny, and M.A. Viergever. Geodesic Deformable Models for Medical Image Analysis. IEEE Trans. Med. Imaging, 17(4):634-641, 1998.

3. S.C. Mitchell, P.F. Lelieveldt, R.J. van der Geest, H.G. Bosch, J.H.C. Reiber, and M. Sonka. Multistage Hybrid Active Appearance Model Matching: Segmentation of Left and Right Ventricles in Cardiac MR Images. IEEE Trans. Med. Imaging, 20(5):415-423, 2001.

4. L.H. Staib and J.S. Duncan. Model-Based Deformable Surface Finding for Medical Images. IEEE Trans. Med. Imaging, 15(5):720-731, 1996.

5. J.A. Schnabel, D. Rueckert, M. Quist, J.M. Blackall, A.D. Castellano-Smith, T. Hartkins, G.P. Penney, W.A. Hall, H. Liu, C.L. Truwit, F.A. Gerritsen, D.L.G. Hill, and D.J. Hawkes. A Generic Framework for Non-rigid Registration Based on Non-uniform Multi-level Free-form Deformations. In Medical Image Computing and Computer Assisted Intervention (MICCAI), pages 573-581, 2001.

6. C. Studholme, D.L.G. Hill, and D.J. Hawkes. An Overlap Invariant Entropy Measure of 3D Medical Image Alignment. Pattern Recognition, 32:71-86, 1999.

7. R.J. van der Geest, V.G.M. Buller, E. Jansen, H.J. Lamb, L.H. Baur, E.E. van der Wall, A. de Roos, and J.H.C. Reiber. Comparison Between Manual and Semiautomated Analysis of Left Ventricular Volume Parameters from Short-Axis MR Images. J. Comput. Assist. Tomogr., 21(5):756-765, 1997. 